\title{
AN INSTRUMENTAL BASIS FOR MULTISPECTRAL LIDAR WITH SPECTRALLY-RESOLVED DISTANCE MEASUREMENTS
}

\author{
D. Salido-Monzú*, A. Wieser
}

Institute of Geodesy and Photogrammetry, ETH Zurich, Switzerland - (david.salido, andreas.wieser)@geod.baug.ethz.ch

\section{Commission II, WG II/3}

KEY WORDS: Hyperspectral and multispectral LiDAR, reflectorless distance measurement, remote material probing, laser scanning, femtosecond laser

\begin{abstract}
:
Hyperspectral solutions augment laser scanning technology with material probing capabilities by measuring target reflectance along with topography. We propose a novel instrumental basis that enables also spectrally-resolved distance measurement with sufficient sensitivity as to access dispersive phenomena on the reflecting target and along the propagation medium, further enhancing the material analysis capabilities of hyperspectral approaches. To this end we have extended distance metrology using intermode beat notes of a mode-locked femtosecond laser to cover flexibly selected spectral regions. The approach is based on an ultra-broadband source derived from a femtosecond laser via coherent supercontinuum generation. Herein we provide a first demonstration of the successful application of this technique to reflectorless measurements and thus its feasibility for multispectral LiDAR. We use a table-top experimental set-up to assess the approach by measuring distance, spectrally-resolved relative distance and reflectance to 5 different material samples on 5 multiplexed contiguous spectral bands of $50 \mathrm{~nm}$ in the range of $600 \mathrm{~nm}$ to $850 \mathrm{~nm}$. We have achieved a distance measurement precision and accuracy better than $100 \mu \mathrm{m}$ using integration times of about $30 \mathrm{~ms}$, with promising perspectives regarding scalability to practical distances. The spectrally-resolved distance measurements also show repeatable material-dependent profiles with differences between materials up to several tenths of $\mathrm{mm}$ in some spectral bands. Combined with simultaneously acquired reflectance estimations, these profiles enable collecting additional target information, indicating the potential of the approach to enhance the material probing capabilities of prospective multispectral laser scanners.
\end{abstract}

\section{INTRODUCTION}

Hyperspectral LiDAR technology has evolved into a promising solution to improve laser scanning by acquiring further information of the reflecting target. In contrast to established quasi-monochromatic systems, hyperspectral approaches provide an additional estimation of the spectral reflectivity of the scanned surface along with its $3 \mathrm{~d}$ topography. This significantly expands the range of possible applications of laser scanning by enabling target material identification (Powers and Davis, 2012) and estimation of additional parameters of interest such as vegetation indices (Chen et al., 2010, Hakala et al., 2012), concentration of certain chemicals (Nevalainen et al., 2013, Du et al., 2016, Sun et al., 2017) or material health indicators (Hemmleb et al., 2006). Current implementations of hyperspectral or multispectral LiDAR typically derive distance and power measurements from locally-referenced delays and amplitudes of ultra-broadband pulsed lasers spectrally split before photodetection (Wang et al., 2018, Kaasalainen et al., 2018). While spectral analysis of the received signals provides radiometric information on the reflecting target, the delay observations for different spectral bands are usually not sensitive enough as to identify dispersive phenomena introduced by the target or propagation medium. It has therefore not been effective so far to analyze individually the delay measurements of the different spectral channels, which are instead usually averaged to enhance the SNR.

In this work, we propose a novel instrumental basis for highly accurate spectrally-resolved distance measurements along with reflectance, providing a promising approach to improve

\footnotetext{
*Corresponding author
}

the material probing capabilities of current multispectral solutions. The proposed technique relies on using the intermode beat notes produced by direct detection of a coherently broadened mode-locked femtosecond laser (fs-laser). This approach provides high quality signals for phase-based distance estimation without explicit intensity modulation of the laser, while enabling measurements over a versatile range of spectral bands.

The basis of the proposed measurement principle is covered in Section 2.1. Section 2.2 describes the experimental set-up developed to assess its feasibility and performance. The results from this assessment are shown and discussed in Section 3 and, finally, the main implications and conclusions of this work are summarized in Section 4.

\section{INSTRUMENTAL BASIS}

\subsection{Measurement principle}

Mode-locked fs-lasers produce trains of ultra-short pulses generated by the periodical interference of thousands of modes resonating in the laser cavity with a fixed phase relationship. This operation principle is reflected in the spectrum of the generated signal, composed by a collection of phase-locked equally spaced spectral lines forming the so-called optical frequency comb (Ye and Cundiff, 2005). Figure 1 shows a schematic plot of such a spectrum, where the amount of lines is reduced for clarity. A typical mode-locked fs-laser's spectrum covers several tens of nm with line spacing defined by the pulse repetition rate $\left(f_{r}\right)$ of a few hundred $\mathrm{MHz}$ up to a few $\mathrm{GHz}$, hence containing hundreds of thousands of spectral lines. 


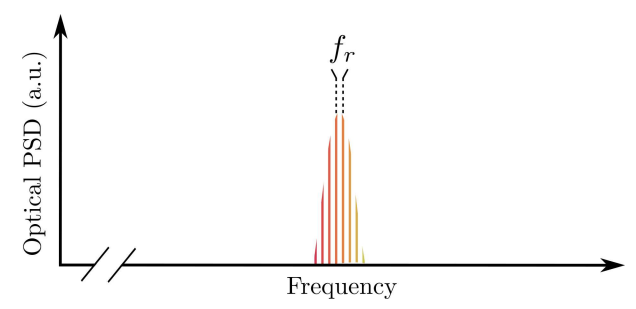

Figure 1. Optical frequency comb of mode-locked fs-laser with repetition rate $f_{r}$ (line density reduced for clarity).

When a fs-laser beam illuminates a square-law detector such as a standard photodiode, self mixing between all possible combinations of the comb modes produces beat notes in the detector's electrical output. These intermode beat notes form an equivalent frequency comb in the generated photocurrent, composed of a series of high quality tones at the integer multiples of $f_{r}$ up to the photodetector's (PD) bandwidth. An example of such a signal is shown in Figure 2.

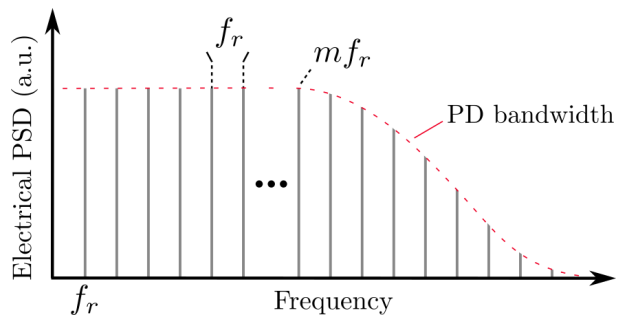

Figure 2. Electrical frequency comb produced by intermode beating upon direct photodetection of a mode-locked fs-laser with repetition rate $f_{r}$.

Each of these tones contains information of the optical group delay and power of the detected beam in its accumulated phase and amplitude, respectively. The phase $\phi$ of the $m_{t h}$ beat note of frequency $m f_{r}$ of a signal detected after propagating over a distance $L$ can be simplified to

$$
\phi\left(m f_{r}\right)=\frac{2 \pi n_{g}\left(f_{c}\right)}{c_{0}} m f_{r} L+\theta_{0},
$$

where $n_{g}\left(f_{c}\right)$ is the integral group refractive index of the medium at the center frequency $f_{c}$ of the laser, $c_{0}$ is the speed of light in vacuum and $\theta_{0}$ is an unknown phase origin. A complete derivation of the related equations can be found in (Salido-Monzú and Wieser, 2018). The phase is proportional to the propagation length and independent of the beat note amplitude. Given sufficient knowledge of the beat frequency and the refractive index, it can thus be used to measure the distance. Elimination of the unknown phase bias and establishing a relation between the electro-optical signal and the mechanics of the instrument is possible through measurement of the phase $\phi_{0}$ of an equivalent beat note generated by the same optical signal in a different detector with fixed path length $L_{0}$. By calculating the difference between the two, the referenced distance $d=L-L_{0}$ is obtained:

$$
d=L-L_{0}=\frac{c_{0}}{2 \pi n_{g}\left(f_{c}\right) m f_{r}}\left[\phi\left(m f_{r}\right)-\phi_{0}\left(m f_{r}\right)\right] .
$$

Similarly, with proper radiometric calibration the amplitude of each beat note can be used to derive the integral attenuation of the optical signal, hence accounting for absorption of the propagation medium and for target reflectance.

The beat notes can be considered equivalent to the sinusoidal intensity modulations on continuous-wave lasers used for phase-based distance estimation in high-end electro-optical distance measurement (EDM) or laser scanning instruments. The intermode beating approach has been demonstrated as a robust alternative to this established technique (Minoshima and Matsumoto, 2000, Minoshima et al., 2006, Doloca et al., 2010, Jang et al., 2014), providing multiple high quality and high bandwidth modulation signals suitable for EDM without using any electrical or optical modulators. This yields lower instrumental errors by reducing crosstalk while enabling flexible multi-frequency measurements that deliver high resolution and high non-ambiguity and enhance mixed pixel detectability. As with EDM systems, the actual phase measurement is obtained modulo $2 \pi$ with intermode beating, and phase ambiguity resolution is necessary for obtaining the full distance with eq. (2).

We have in the past extended this approach to an ultra-broadband source derived from a fs-laser through coherent supercontinuum generation (SCG). This technology relies on a collection of non-linear processes occurring within a microstructured photonic-crystal fiber (PCF) to produce severe spectral broadening of the seed laser while preserving its phase coherence (Dudley et al., 2006). By using the intermode beating approach on filtered bands of the coherent supercontinuum, our proposed solution enables accurate distance measurements over several flexibly selected spectral bands. We developed a simultaneous dual-band table-top demonstrator of the method (Salido-Monzú and Wieser, 2018, Salido-Monzú and Wieser, 2017), showing that it may enable inline correction of atmospheric delays by exploiting atmospheric dispersion. The feasibility to obtain highly accurate multispectral distance estimates was thus demonstrated on a cooperative target (corner cube prism) returning most of the optical power. In this work, we extend the scope of the method by applying it to reflectorless measurements and multiple spectral bands to prove its potential applicability to material probing.

Fs-laser supercontinua can cover several hundred nm, whereas a band of only a few $\mathrm{nm}$ is necessary to generate high quality beat notes. There is therefore potential to accommodate many spectral bands along the spectrum. These can be selected from the backreflected signal by either time-multiplexing spectral filters or a diffraction grating on a single photodetector or, if simultaneity is needed, by implementing parallel photodetection with as many stages as desired channels. The selection of a specific design depends on the trade-off between required measurement dynamics, spectral resolution and implementation complexity.

A general, simplified diagram of the proposed instrumental basis assuming simultaneous detection is shown in Figure 3. A fs-laser whose repetition rate $f_{r}$ is locked to a frequency standard, e.g. a rubidium atomic clock, is pumped into the PCF of a supercontinuum generation stage to produce the coherent ultra-broadband beam. After acquiring a portion of the beam for delay and power reference, this is launched towards the target. Scanning capabilities can be incorporated with a standard design using a planar or parabolic mirror mounted on an automated 2-axis gimbal. A telescope co-aligned with the launching stage collects part of the diffused backreflection, and directs it to the detection stage, where it is spectrally separated in the bands of interest before photodetection. 


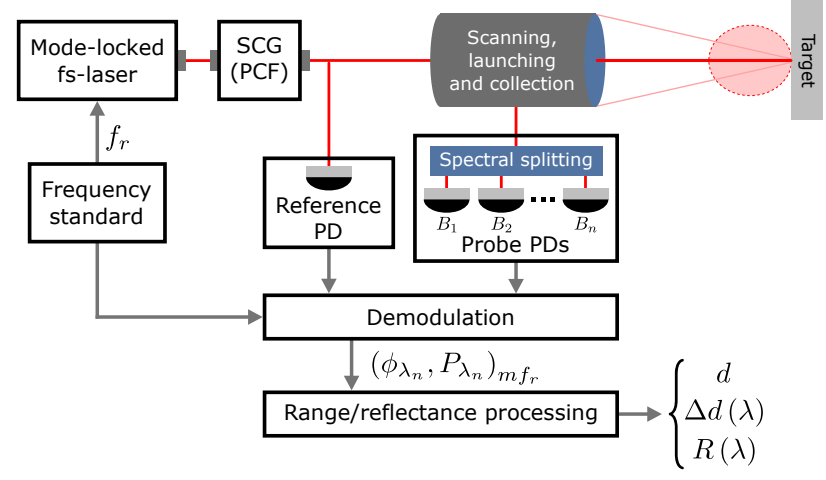

Figure 3. Diagram of basic operation principle.

An example of the spectral selection on a realistic supercontinuum is depicted in Figure 4. The spectral inhomogeneity characteristic to broadened fs-lasers has to be accounted for via internal reference measurements and independent gain regulation for each channel. When illuminating the corresponding detector, each band $B_{n}$ produces a series of intermode beat notes whose phase and amplitude are respectively proportional to the group delay and optical attenuation accumulated on the corresponding spectral band. These can then be easily extracted from each beat note by synchronously demodulating them including the signals from the reference detector and the frequency standard.

The complete measurement process results in a collection of observed parameters as shown in (3). The multiple measurements for each band, represented in the rows in (3), can be then used to perform a multi-frequency estimation of the absolute range $d$ with high resolution and non-ambiguity, whereas the wavelength-dependent measurements represented columnwise provide the basis to derive reflectance $R(\lambda)$ and multispectral relative distance $\Delta d(\lambda)$ estimations. While eq. (3) shows the complete set of possible observed parameters, in practice (and in the results shown later in this paper) only a subset of these measurements will actually be calculated and output by the instrument depending on the range and spectral requirements of the application.

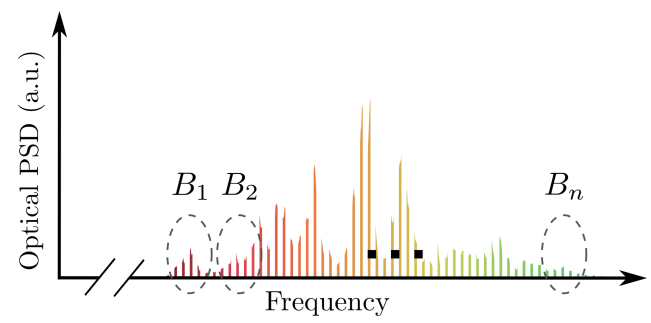

Figure 4. Diagram of supercontinuum spectrum with selected bands.

$$
\begin{array}{cc}
B_{1}: & \left(\phi_{\lambda_{1}}, P_{\lambda_{1}}\right)_{f_{r}}, \cdots,\left(\phi_{\lambda_{1}}, P_{\lambda_{1}}\right)_{m f_{r}} \\
B_{2}: & \left(\phi_{\lambda_{2}}, P_{\lambda_{2}}\right)_{f_{r}}, \cdots,\left(\phi_{\lambda_{2}}, P_{\lambda_{2}}\right)_{m f_{r}} \\
\vdots & \\
B_{n}: & \left(\phi_{\lambda_{n}}, P_{\lambda_{n}}\right)_{f_{r}}, \cdots,\left(\phi_{\lambda_{n}}, P_{\lambda_{n}}\right)_{m f_{r}}
\end{array}
$$

\subsection{Experimental set-up}

We have developed a table-top experimental set-up based on a commercial fs-laser and SCG module (MenloSystems C-Fiber 780 + SCG1500) to proof the fundamental feasibility of performing reflectorless measurements with the proposed approach, and assess the sensitivity of the multispectral relative distance and reflectance estimates. We have used this set-up to probe the distance and reflectance of samples from various common materials. The measurements are derived from the phase and amplitude of the $1 \mathrm{GHz}$ intermode beat notes (corresponding to phase-based distance measurement with a $30 \mathrm{~cm}$ modulation wavelength) produced upon detection of spectral portions of the backreflected supercontinuum, referenced to equivalent beat notes acquired on a fixed reference path. Each distance and power measurement is carried out on 5 contiguous spectral bands of $50 \mathrm{~nm}$ in the visible and near-infrared regions in the range of $600 \mathrm{~nm}$ to $850 \mathrm{~nm}$, selected by sequentially filtering the broadband source. Figure 5 shows an actual acquisition of our available supercontinuum, depicting the 5 spectral bands used herein.

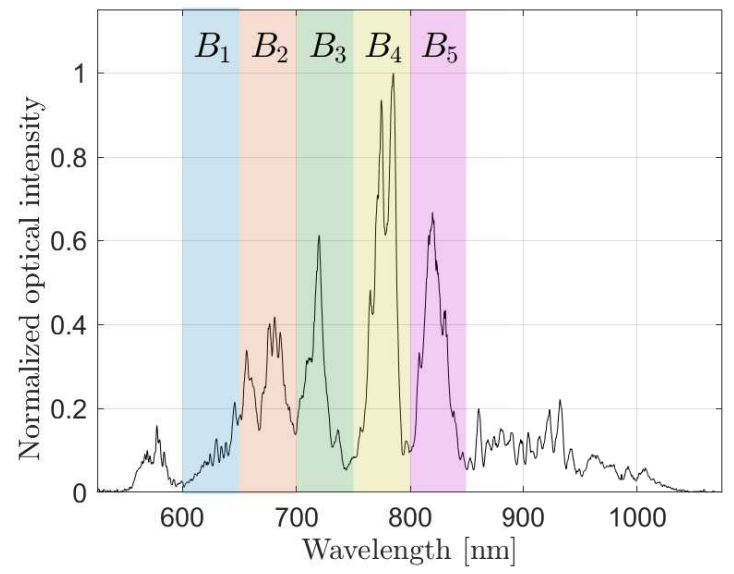

Figure 5. Supercontinuum spectrum and filtered spectral bands used in the experimental set-up.

After low-level conditioning and downmixing to an intermediate frequency, the beat notes acquired for each band are digitized and their phase and power extracted through synchronous I/Q demodulation. In addition to the phase reference path, the set-up includes an automated bypass of the delay line for fast self-reference measurements compensating drifts in the complete measuring chain of the probe detector.

We have used this set-up to probe 5 different material samples (untreated and varnished medium-density fiberboard (MDF) wood, aluminum, concrete and gypsum) displaced along a $50 \mathrm{~mm}$ delay line and continuously referenced to multiplexed measurements on a diffuse reflectance standard (Spectralon $60 \%$ ) at a fixed position. The delay line is implemented with a calibrated motorized translation stage whose internal position feedback is used as distance reference. A picture of the optical stage of this experimental set-up is shown in Figure 6. Detailed descriptions of both optical and electronic stages are given in (Salido-Monzú and Wieser, 2019).

\section{RESULTS}

The experimental set-up was firstly used to assess the fundamental feasibility of performing reflectorless distance 


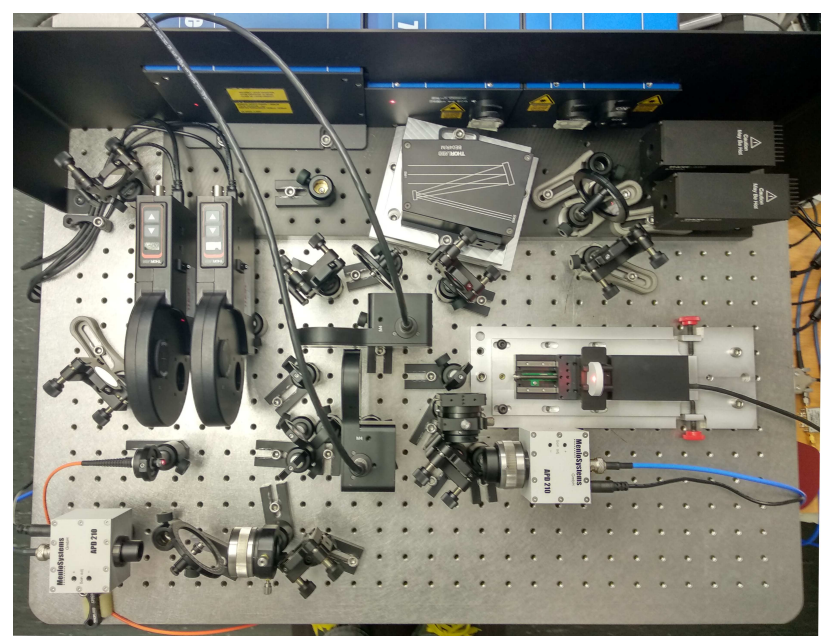

Figure 6. Optical set-up with Spectralon target on the delay line.

measurements with the proposed approach. Discussing the achieved absolute distance measurement performance is not the goal of this manuscript, whose focus is mainly on the capabilities of the proposal to spectrally resolve material dependent relative distances. Detailed information on the distance performance evaluation is given in (Salido-Monzú and Wieser, 2019), while only a summary of the main results is provided herein for completeness. Long term stability and precision have been evaluated on static measurements on the Spectralon target on a fixed position over more than 5 hours, yielding standard deviations better than $100 \mu \mathrm{m}$ on all bands using integration times of about $30 \mathrm{~ms}$. Distance measurement accuracy has been evaluated also on the Spectralon target on $1 \mathrm{~mm}$ steps along the $50 \mathrm{~mm}$ of the delay line, yielding an accuracy better than $50 \mu \mathrm{m}$ after calibration of cyclic errors mainly caused by optical multipath and focus variation with distance. We believe that this is a promising reflectorless performance, whose scalability to practical distances over several tens of meters can be achieved with a more efficient launching and detection stage, using larger integration times and accessing higher beat notes through faster photodetectors, none of which presents any expected critical challenges.

Using the Spectralon as a reference for the distance origin at each band, we have measured the relative multispectral distances on all the material samples placed at the same fixed distance. Figure 7 shows the results for untreated wood. The measurements have been carried out on 4 different points of the sample in order to get a first impression of variability within the target specimen (a more detailed investigation is left for future work). Each point has been measured on two independent cycles using an integration time of about $30 \mathrm{~ms}$ per spectral band. The results show a clear pattern in the measured distances, with higher spectral bands yielding smaller distance in relation to the reference target. The differences between the bands are up to about $1 \mathrm{~mm}$, while the range between the different points (but for a single band) is less than $0.2 \mathrm{~mm}$ for all points. The differences within each band suggest that there is a systematic bias between different points of the same sample independent of the measurement band. It is likely caused by insufficient repeatability in the sample repositioning for measuring the different points. Similar measurements have been performed on the other material samples, repeating the complete measurement cycle for all bands 3 times on 1 point per sample. Figure 8 shows the resulting relative distances as a function of material and band, where the average and standard deviation of the measurements on each material and band are depicted by the error bars.

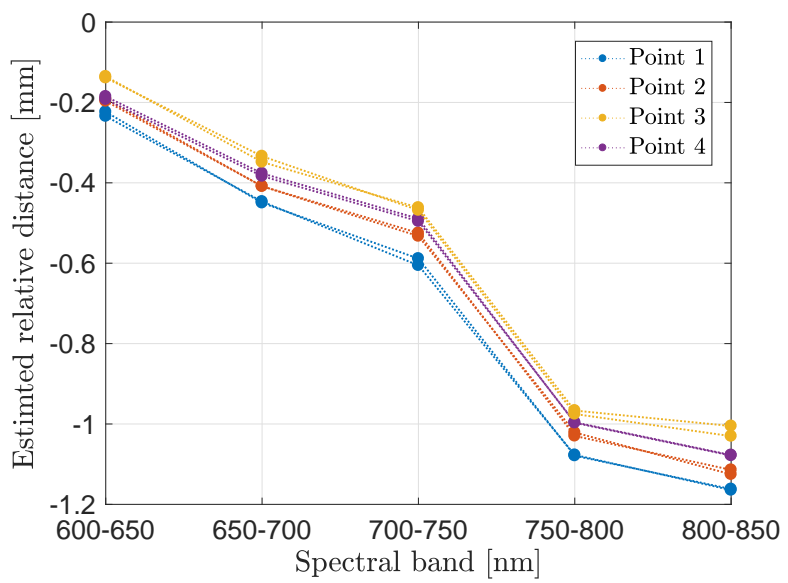

Figure 7. Estimated multispectral distance relative to Spectralon for untreated MDF wood.

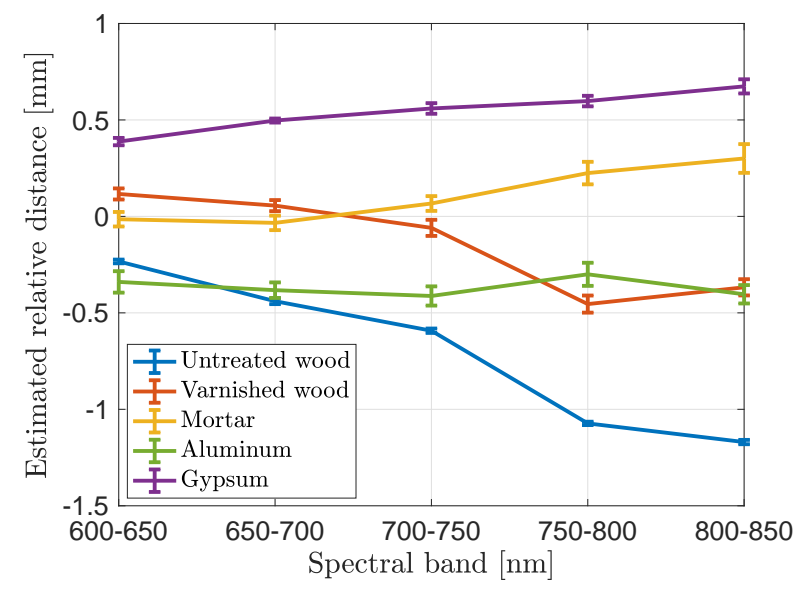

Figure 8. Estimated multispectral distance relative to Spectralon for various materials.

The results show repeatable material-dependent profiles with signatures on the level of several tenths of $\mathrm{mm}$. The average offset between materials is partly caused by variations of the distance between each sample and the Spectralon again due to lack of repeatability of the sample holder. However, the trends contain material-dependent differences significantly larger than the sensitivity of the measurements and the potential inadequacies of this first experimental setup. With proper reference data, these profiles could be therefore used to identify the target material. The differences between materials for specific spectral bands agree with the error magnitudes identified in previous investigations on material-dependent uncertainties in reflectorless EDM (Zámečníková et al., 2014), signaling also that this approach holds potential to mitigate material-related uncertainties by accessing multispectral distance information unattainable for standard instruments. We have not yet investigated which are the dominant physical phenomena causing the observed distance dependencies with wavelength and material. Potential candidates include partial sub-surface penetration of the optical signal defining slightly different effective reflection planes for 
different wavelengths, or dispersive phase shifting of the optical modes - and hence the resulting intermode beat notes-upon reflection. A detailed study and specific investigation on possible causes is outlined for future work.

The potential of the method to also provide reflectance probing capabilities equivalent to those of current hyperspectral laser scanners has been preliminarily tested. Received optical power is derived from the amplitude of the intermode beat notes extracted from each spectral band, and relative reflectance to multiplexed measurements on the Spectralon target is calculated therefrom. Figure 9 shows the estimated reflectance values on normal incidence for the different material samples.

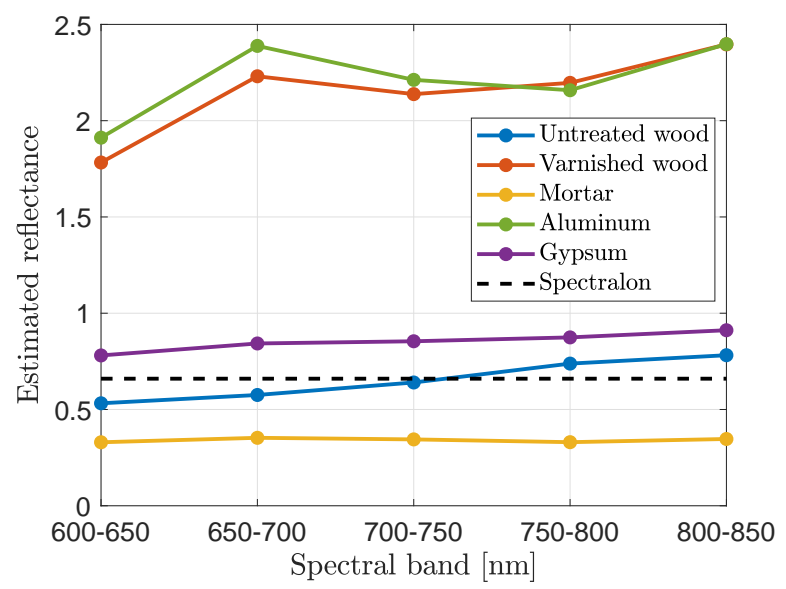

Figure 9. Estimated reflectance at normal incidence on the different spectral bands for various materials.

These reflectance estimates have not been yet validated with reference data. A comparison with independent measurements from a commercial active reflectance spectrometer will be shortly undertaken. Current results are therefore not significant regarding reflectance estimation accuracy, and only provide an initial test for plausibility. The rank of the estimated values agrees with the apparent reflectance of the different materials by visual inspection. Additionally, the aluminum and varnished wood samples yielding normal reflectance levels higher than $100 \%$ is also coherent since those are the only two samples exhibiting a glossy surface finish, indicating strong specular components in their reflection patterns. This can be also observed in Figure 10, where results from measurements of the reflectance as a function of angle of incidence for aluminum and untreated MDF wood, representing a typical glossy and mate material respectively, are depicted. The theoretical Lambertian pattern for the Spectralon target is also shown for reference. Deviations from this pattern for the wood sample are likely caused by changes in the optical coupling efficiency of the experimental set-up as a function of angle of incidence, rather than by an actual non-Lambertian behavior of the specimen surface.

\section{CONCLUSIONS}

We investigate the feasibility of using the intermode beat notes produced by direct detection of a coherently broadened fs-laser to measure spectrally-resolved relative distances along with absolute distance and reflectance on natural targets. The assessment of the proposal on a table-top experimental

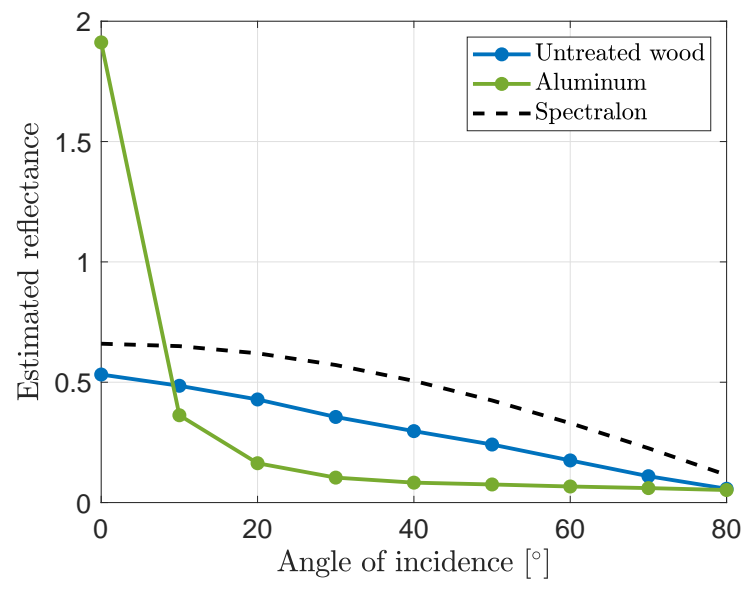

Figure 10. Estimated reflectance at $600-650 \mathrm{~nm}$ as a function of angle of incidence for aluminum and untreated MDF wood.

set-up yielded a promising performance regarding absolute distance accuracy and precision, with positive perspectives for its scalability to practically useful ranges. The results also show that the approach offers sufficient delay sensitivity in the individual spectral channels as to detect dispersive effects on the reflecting material and potentially also the propagation medium. This allows access to additional material parameters-such as wavelength-dependent surface penetration-and, when measuring on well-known geometries, may enable partial estimation of the integral composition or state of the atmosphere along the propagation path. Moreover, the combination of these spectrally-resolved distance measurements with reflectance estimations from simultaneously acquired multispectral power signatures offers further potential to collect additional target information. We see the proposed approach as a promising instrumental basis for prospective multispectral LiDAR solutions with enhanced material and medium probing capabilities.

\section{REFERENCES}

Chen, Y., Räikkönen, E., Kaasalainen, S., Suomalainen, J., Hakala, T., Hyyppä, J. and Chen, R., 2010. Two-channel hyperspectral LiDAR with a supercontinuum laser source. Sensors 10(7), pp. 7057-7066.

Doloca, N. R., Meiners-Hagen, K., Wedde, M., Pollinger, F. and Abou-Zeid, A., 2010. Absolute distance measurement system using a femtosecond laser as a modulator. Measurement Science and Technology 21(11), pp. 115302-115308.

Du, L., Gong, W., Shi, S., Yang, J., Sun, J., Zhu, B. and Song, S., 2016. Estimation of rice leaf nitrogen contents based on hyperspectral LIDAR. International Journal of Applied Earth Observation and Geoinformation 44, pp. 136-143.

Dudley, J. M., Coen, S. and Genty, G., 2006. Supercontinuum generation in photonic crystal fiber. Reviews of Modern Physics 78(4), pp. 1135-1184.

Hakala, T., Suomalainen, J., Kaasalainen, S. and Chen, Y., 2012. Full waveform hyperspectral LiDAR for terrestrial laser scanning. Optics Express 20(7), pp. 422-430. 
Hemmleb, M., Weritz, F., Schiemenz, A., Grote, A. and Mairhofer, C., 2006. Multi-spectral data acquisition and processing techniques for damage detection on building surfaces. In: Archives of 2006 ISPRS Commission V Symposium, pp. 1-6.

Jang, Y.-S., Lee, K., Han, S., Lee, J., Kim, Y.-J. and Kim, S.-W., 2014. Absolute distance measurement using the frequency comb of a femtosecond laser. Optical Engineering 53(12), pp. 122403-122408.

Kaasalainen, S., Malkamaki, T., Ilinea, J. and Ruotsalainen, L., 2018. Multispectral Terrestrial Laser Scanning: New Developments and Applications. In: Proc. of the 2018 IEEE International Geoscience and Remote Sensing Symposium, IEEE, pp. 8737-8740.

Minoshima, K. and Matsumoto, H., 2000. High-accuracy measurement of 240-m distance in an optical tunnel by use of a compact femtosecond laser. Applied Optics 39(30), pp. 5512-5517.

Minoshima, K., Schibli, T. R., Matsumoto, H., Iino, Y., Yoshino, K. and Kumagai, K., 2006. Ultrahigh dynamic-range portable distance meter using an optical frequency comb. In: Proc. of the 2006 Conference on Lasers and Electro-Optics and 2006 Quantum Electronics and Laser Science Conference, pp. 3-4.

Nevalainen, O., Hakala, T., Suomalainen, J. and Kaasalainen, S., 2013. Nitrogen concentration estimation with hyperspectral lidar. In: ISPRS Annals of Photogrammetry, Remote Sensing and Spatial Information Sciences, Vol. II-5/W2, pp. 205-210.

Powers, M. A. and Davis, C. C., 2012. Spectral LADAR: active range-resolved three-dimensional imaging spectroscopy. Applied Optics 51(10), pp. 1468-1478.

Salido-Monzú, D. and Wieser, A., 2017. A new approach to long distance EDM : Using intermode beating of broadband ultrashort laser pulses. In: Proc. of the 2017 International Conference on Engineering Surveying, pp. 173-182.

Salido-Monzú, D. and Wieser, A., 2018. Simultaneous distance measurement at multiple wavelengths using the intermode beats from a femtosecond laser coherent supercontinuum. Optical Engineering 57(4), pp. 044107-044116.

Salido-Monzú, D. and Wieser, A., 2019. Multispectral reflectorless distance measurement using a femtosecond laser coherent supercontinuum. In: Proc of the 2019 SPIE Optical Metrology, (accepted).

Sun, J., Shi, S., Gong, W., Yang, J., Du, L., Song, S., Chen, B. and Zhang, Z., 2017. Evaluation of hyperspectral LiDAR for monitoring rice leaf nitrogen by comparison with multispectral LiDAR and passive spectrometer. Scientific Reports 7, pp. 1-9.

Wang, Z., Chen, Y., Li, C., Tian, M., Zhou, M., He, W., Wu, H., Zhang, H., Tang, L., Wang, Y., Zhou, H., Puttonen, E. and Hyyppa, J., 2018. A Hyperspectral LiDAR with Eight Channels Covering from VIS to SWIR. In: Proc. of the 2018 IEEE International Geoscience and Remote Sensing Symposium, IEEE, pp. 4293-4296.

Ye, J. and Cundiff, S. T., 2005. Femtosecond optical frequency comb: principle, operation and applications. Springer.
Zámečníková, M., Wieser, A., Woschitz, H. and Ressl, C., 2014. Influence of surface reflectivity on reflectorless electronic distance measurement and terrestrial laser scanning. Journal of Applied Geodesy 8(4), pp. 311-325. 